\title{
De Pronasol a la Cruzada. ¿Qué hay de nuevo sobre coordinación?
}

\section{From Pronasol to Cruzada. What is new about coordination?}

doi: http://dx.doi.org/10.32870/

espiral.v25i71.6179

\section{Resumen}

En este trabajo, se analizan y comparan los mecanismos e instrumentos de coordinación entre el Programa Nacional Solidaridad y la Cruzada Nacional contra el Hambre, a través de un análisis documental que permite mostrar similitudes y diferencias. Se observa que la búsqueda de la coordinación intergubernamental e interinstitucional es un requisito de difícil consecución en la política social. Este trabajo aporta elementos de análisis para la política social y la administración pública.

Palabras clave: política social, coordinación intersectorial, planificación de programas, programa Solidaridad, Cruzada Nacional contra el Hambre.

\author{
Verónica Martínez-Flores \\ Guillermina Benavides Rincón**
}

\begin{abstract}
This paper aims to analyse and compare the mechanisms used to promote coordination in the Programa Nacional de Solidaridad (National Solidarity Program) and the Cruzada Nacional contra el Hambre (National Crusade against Hunger). Document analysis is employed to identify similarities and differences between the two programs. The results show that intergovernmental and interinstitutional coordination, although a desirable outcome, has always been difficult to achieve in social policy. This research provides elements for the analysis of social policy and public administration.
\end{abstract}

Keywords: Social policy, intersectoral cooperation, programme planning, Solidaridad program, Cruzada Nacional contra el Hambre.

-Profesora-Investigadora del Instituto de Ciencias Sociales y Administración, Universidad Autónoma de Ciudad Juárez (UACJ), México. ORCID: http://orcid.org/0000-0003-3999-2003. veronica.martinez@uacj.mx

- Profesora-Investigadora de la Escuela de Gobierno y Transformación Pública, Instituto Tecnológico y de Estudios Superiores de Monterrey (ITESM), México. ORCID: http://orcid. org/0000-0002-2014-3848.

gbr@itesm.mx

Fecha de recepción: 02 de octubre de 2016. Fecha de aceptación: 17 de julio de 2017. 


\section{Introducción}

La dispersión de políticas y programas en materia de desarrollo social ha llevado a los Gobiernos a incorporar en el diseño de las políticas públicas esquemas de coordinación y colaboración. La coordinación se vuelve particularmente importante ante problemas de amplio impacto como la pobreza y la falta de atención a los derechos sociales de una población, dada la existente incapacidad para dar respuesta a estas problemáticas a partir de una sola secretaría o entidad gubernamental ante la multidimensionalidad de los fenómenos.

En México, desde el lanzamiento del Programa de Inversiones para el Desarrollo Rural (PIDER), implementado de 1970 a 1982, se incorporó la coordinación con el objetivo de sumar esfuerzos para la atención de la pobreza. De acuerdo a Barajas, "el PIDER fue el primer mecanismo administrativo en la historia de México que basó su operación en una coordinación y cooperación de las dependencias federales y estatales" (2002, p. 70). Desde entonces, la búsqueda de coordinación se ha mantenido en los programas y estrategias de política social. Uno de los programas emblemáticos, el Programa Nacional Solidaridad (Pronasol), establecía la coordinación como un requisito de la política social, al igual que la integralidad de la misma.

En la actualidad, también se plantea la coordinación como parte importante de la Cruzada Nacional contra el Hambre ( $\mathrm{CNCH})$ : dentro del Programa Nacional México sin Hambre 2014-2018, que enmarca a la $\mathrm{CNCH}$, se le reconoce como el segundo eje del "nuevo enfoque de atención", aunque se distingue entre coordinación interinstitucional y coordinación intergubernamental, la primera al interior del Gobierno federal y la segunda entre la federación, los estados y municipios, incluyendo en esta última mecanismos 
de participación ciudadana (Diario Oficial de la Federación, 2014).

De esta manera, se vuelve evidente que la búsqueda de la coordinación no es un tema reciente en la política social mexicana. El objetivo de este trabajo es analizar y comparar los mecanismos e instrumentos de coordinación del Pronasol y la $\mathrm{CNCH}$, dada la similitud de diseño y alcance de ambas estrategias de política social.

Para lograr esto, se realizó un análisis de contenido de diferentes documentos y reportes de las políticas estudiadas. En el primer apartado, se hace una revisión de la política social en México, así como de las principales propuestas en temas de coordinación de la política pública. Posteriormente, se describen los casos de Pronasol y CNCH. Finalmente, se presentan el estudio comparativo sobre la coordinación y las conclusiones.

\section{Política social en México}

La política social es parte central de un modelo de desarrollo. En ella, se encuentran las concepciones y acciones de un esquema de desarrollo social y económico, pero también de un modelo político. En el caso de México, la Constitución de 1917 otorgó el derecho a la seguridad social, la educación, los servicios de salud y la vivienda (Brachet Márquez, 2004), pero no fue hasta las siguientes décadas que el Estado mexicano empezó a formalizar muchos de estos derechos, especialmente a partir de 1943 con la creación de la Ley del Seguro Social.

Si bien la intención de esta ley era establecer un sistema de bienestar universal, esto nunca se logró, ya que la afiliación a la seguridad social fue condicionada al empleo formal, dejando a importantes sectores de la población, principalmente campesinos y trabajadores urbanos informales, fuera de los beneficios de protección social (Barba, 2004). Para 
algunos autores, la formalización de la seguridad social, a partir de la aprobación de la Ley del Seguro Social y la creación del Instituto Mexicano del Seguro Social (IMSS), también en 1943, respondió a la necesidad de industrialización del país (Brachet Márquez, 2004; Ordóñez Barba, 2002; Vilas, 2000).

Es decir, a pesar de que el IMSs se diseñó para proveer una amplia gama de servicios sociales, tales como seguro médico, de incapacidad, de trabajo y de vida, servicio de guardería, acceso a áreas deportivas y culturales, así como pensiones y préstamos para vivienda, un amplio sector de la población mexicana no tenía acceso a la seguridad social pues no contaba con un trabajo formal (Levy, 2008). Como era de esperarse, tal situación tuvo un impacto directo en los niveles de bienestar de la población, especialmente en el sector rural. Así, para contrarrestar los efectos adversos, el Estado empezó a ofrecer subsidios universales a alimentos básicos y programas de asistencia social. Sin embargo, muchos de estos subsidios y programas llegaron sólo a la población de las zonas urbanas, ampliando la brecha entre el sector rural y urbano (Levy, 1994; Ordóñez Barba, 2002).

En este contexto, a partir de los años setenta, diferentes programas antipobreza fueron implementados en México con el objetivo de atender a los pobres rurales que habían sufrido el abandono del Gobierno. Ordóñez Barba (2002) identifica dos periodos en las estrategias de combate a la pobreza en el país: el primero durante los años setenta y ochenta, cuando hubo una reorientación del gasto de gobierno hacia las áreas rurales para promover su desarrollo, dado que el Gobierno pensaba que los pobres urbanos eran una manifestación de los pobres rurales; y el segundo iniciado a finales de la década de los ochenta, cuando se buscó un nuevo equilibrio entre el Gobierno y el mercado como proveedores de bienestar (Ordóñez Barba, 2002). 
El cambio en el modelo de desarrollo mexicano, impulsado en los años ochenta, fomentó un nuevo esquema en la política social y trajo consigo una nueva estructura institucional para el desarrollo social. Para finales de dicha década, se impulsó un esquema de focalización que dio paso a programas como Solidaridad y el Programa de Educación, Salud y Alimentación (Progresa-Oportunidades) dentro del contexto de esquemas más amplios denominados estrategias de política social. En el sexenio de Vicente Fox (2000-2006), al programa producto de este esquema de focalización se le denominó Contigo; en el periodo de Felipe Calderón (2006-2012), Vivir Mejor; mientras que en la actualidad, en la presidencia de Enrique Peña Nieto (2012-2018), se implementó la ya mencionada CNCH.

Desde el lanzamiento del Pronasol, el discurso del Gobierno ha resaltado la necesidad de coordinación e integralidad de la política social, posicionando incluso a la coordinación como eje central de esta última. Así, por ejemplo, entre las acciones que el Estado realiza para mejorar el bienestar, individual o colectivo, se incluyen algunas llevadas a cabo por los tres niveles de Gobierno (Levy y Rodríguez, 2005), y desde sus diferentes instituciones. No obstante, como ya se ha dicho, la búsqueda de la coordinación ha sido una constante desde el primer programa de combate a la pobreza en México.

\section{2. ¿Coordinación o colaboración? Hacia la integralidad de las políticas}

Al hacer una revisión en torno a los conceptos de coordinación y colaboración, se observa que existe una confusión y, por lo tanto, falta de consenso respecto a estos conceptos. Peters (1998) define la coordinación como "el resultado de un proceso donde las políticas y los programas de gobierno se caracterizan por niveles mínimos de redundancia, incohe- 
rencias y vacíos" (p. 296). Para Rogers y Whetten (1982), en ella participan diversas organizaciones quienes hacen uso de las reglas existentes y a partir de las cuales negocian colectivamente. Por su parte, Wood y Gray (1991) reconocen que la colaboración se presenta "cuando un grupo de actores autónomos de un tema o problema específico participan en un proceso interactivo, utilizando reglas, normas y estructuras compartidas, para actuar o tomar decisiones en temas relacionados a ese problema en particular" (p. 146). ${ }^{1}$

La definición ofrecida por estos autores, y retomada por otros más (Ansell y Gash, 2008; Bryson, Crosby y Stone, 2006; O'Leary y Vij, 2012), se refiere a aquella colaboración que surge del interés propio de los actores por incrementar el valor público de los servicios ofrecidos. Los actores, o stakeholders, pueden ser pero no están limitados a entidades de Gobierno, sin embargo, el énfasis de la gestión pública colaborativa es la participación de actores fuera del sector público, como en la sociedad civil y el sector privado. Así, el enfoque del análisis de Wood y Gray (1991) está dirigido a identificar las fases del proceso de colaboración y la importancia de la función que desempeña el coordinador o líder de la colaboración (Gray, 1989).

De esta manera, el tipo de actores que participan en el proceso de una política pública se presenta como una distinción entre colaboración y coordinación pues, de acuerdo a Peters (2015) y a Bouckaert, Peters y Verhoest (2010), esta última hace referencia principalmente a la intervención de los diferentes actores gubernamentales, por lo que el análisis respecto a la coordinación se enfoca en observar los incentivos impuestos o creados para lograr la coordinación de los planes, políticas y programas al interior de la propia administración del Gobierno. 
Sin embargo, el mismo Peters (2015), al identificar una serie de dimensiones de análisis para la coordinación dentro del sector público, introduce también a la sociedad civil como parte del proceso de coordinación, como se observa en sus siguientes dimensiones:

- Positiva o negativa: la coordinación negativa sucede cuando los actores acuerdan no afectar la operación de otro actor. Su objetivo es minimizar el conflicto. La coordinación positiva, por el contrario, sucede cuando los programas o las organizaciones trabajan juntos para lograr objetivos que no se podrían lograr a través de su acción individual, por lo que actuar de forma coordinada resulta útil.

- Como proceso o resultado: si el objetivo planteado para la coordinación es eliminar las duplicidades de los planes, políticas y programas, es una concepción de coordinación por resultados, pero si hace referencia al trabajo colaborativo para diseñar mejores políticas públicas, se trata de una coordinación desde el proceso.

- Vertical u horizontal: la coordinación horizontal es aquella que se refiere a los planes, programas y dependencias de un mismo nivel de Gobierno, mientras que la que se presenta entre niveles de Gobierno es la que se refiere como vertical.

- Desde el diseño o la implementación: la que se presenta en el diseño de la política pública tiene un enfoque de arriba-abajo e infiere que el diseño debe de ser lo suficientemente bueno para que la implementación sea viable y coordinada. La coordinación desde la implementación tiene un enfoque de abajo-arriba, y parte de la base de que los implementadores tienen margen de maniobra para poder coordinarse en la operación de la política pública. 
- Hacia adentro o afuera: esta dimensión contempla aquella coordinación que se da con actores externos al sector público, como la sociedad civil y el sector privado.

- Con objetivos similares o complementarios: la primera se da cuando los programas atienden el mismo problema público, mientras la segunda cuando estos se complementan.

- Con objetivos específicos o sistémicos: esta dimensión diferencia aquellos objetivos que no son específicos de programas o acciones sociales en particular de objetivos transversales como la equidad de género o la mejora del medio ambiente, y de objetivos sistémicos de todo el aparato gubernamental que no necesariamente cuentan con programas específicos para lograrlos.

- Corto o largo plazo: se habla de la coordinación de corto plazo cuando se presenta en un proyecto en específico o si se volvió necesaria por algún desastre natural cuya respuesta y atención implica la coordinación de diferentes niveles y oficinas de Gobierno que, al pasar la contingencia, volverán a trabajar de manera aislada; por el contrario, cuando se busca institucionalización, entonces su objetivo es a largo plazo.

La participación de los actores externos al sector público se presenta en la coordinación hacia afuera. En ella, se puede reconocer lo que Gray (1989) define como colaboración o esquema de gobernanza colaborativa. Por su parte, Peters (2015) reconoce que, para disminuir las inconsistencias y las duplicidades en la política pública, la coordinación debe darse desde el proceso y no sólo en el resultado.

En torno a los objetivos, es importante mencionar que, de acuerdo con Peters (2015), no necesariamente la coordinación es más sencilla cuando la política pública o tema se encuentra en la misma línea, ya que intentar coordinar programas que atienden el mismo problema público en 
algunas ocasiones dificulta la coordinación, pues los programas compiten por los mismos recursos escasos al tener objetivos similares. El caso contrario sucede cuando los objetivos de los programas son complementarios. En este sentido, cuando cada programa tiene un objetivo específico y diferenciado, la coordinación suele ser más sencilla porque los programas no se perciben como competidores.

En general, cada una de estas dimensiones de análisis identificadas por Peters (2015) utiliza los marcos de análisis para la coordinación: los mercados, las redes y la jerarquía (Alexander, 1995; Bouckaert, Peters y Verhoest, 2010; Thompson y Open University, 1991). Es importante señalar que el uso de estos enfoques difícilmente se dará de forma aislada y, en la mayoría de los casos, se utilizará una combinación dependiendo en gran medida de cada caso en particular. La Tabla 1 resume la descripción de estos enfoques de análisis para la coordinación.

Asimismo, cada uno de estos esquemas utiliza los mecanismos básicos para la negociación política, tales como la negociación, la autoridad, la cooptación y la coerción. La elección de los mecanismos para coordinarse dependerá de factores como la interdependencia de los actores, el tipo de problema para el cual se requiere coordinación y la fase en el proceso de la política pública en donde se busque la coordinación, ya sea en el diseño de la política pública o en su implementación.

Es importante señalar que la coordinación tiene un alto costo, por lo que tendrá sentido buscar coordinarse sólo en aquellos temas o problemas en donde un solo actor no pueda resolver el problema (Bryson, Crosby y Stone, 2006; Peters, 2015; Thomson y Perry, 2006). Por esto, la mayor parte de los casos en donde se busca y es deseable la coordinación es en aquellos problemas complejos (denominados en inglés como wicked problems) para los que una secretaría de Gobierno no tiene la solución, sino que se tienen que 


\section{abordar a partir de una visión holística (Bryson, Crosby y Stone, 2006; Cunnill Grau, 2014).}

\section{Tabla 1. Enfoques en el análisis de coordinación}

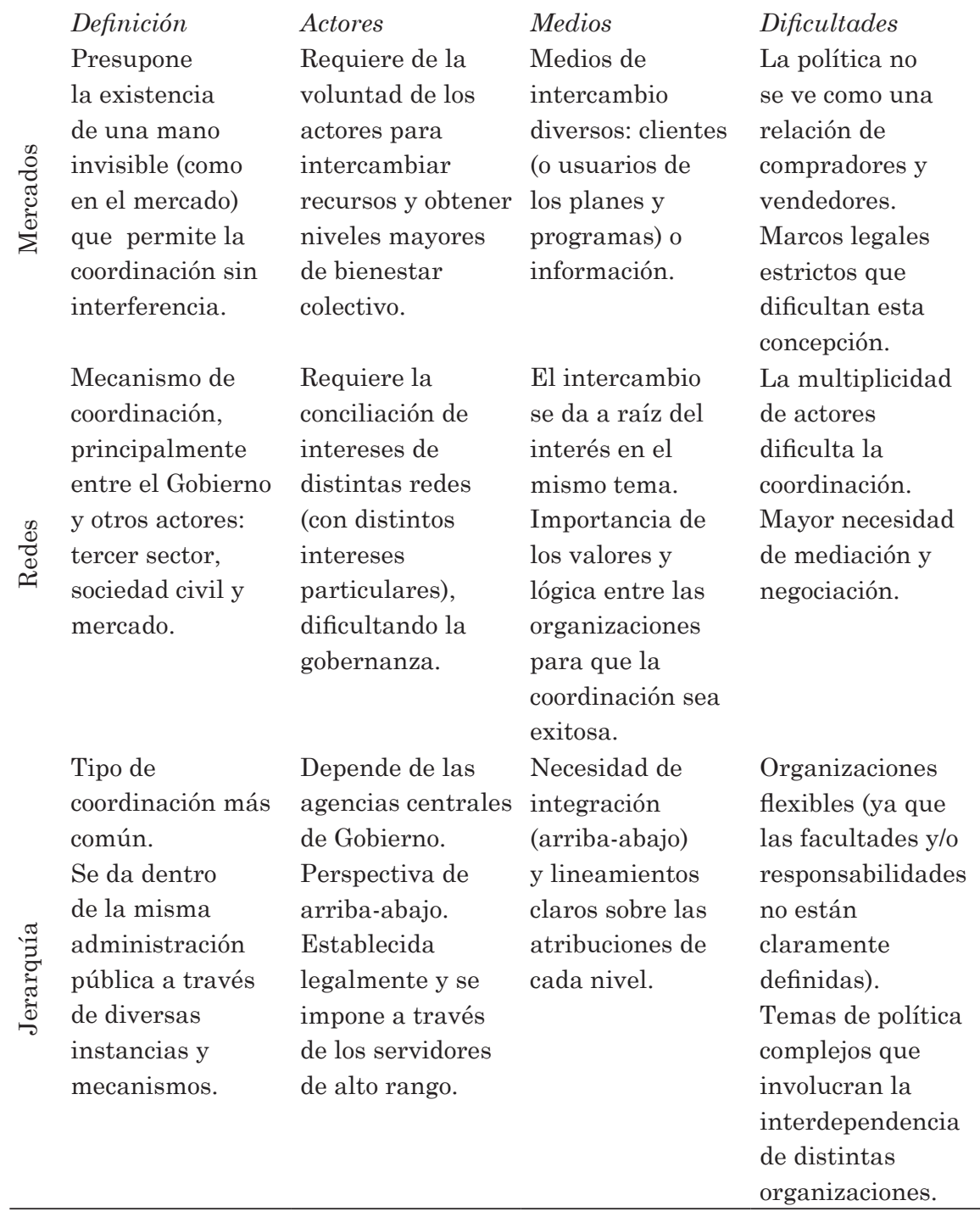

Fuente: elaboración propia con información de Peters (1998 y 2005). 
Sobre la colaboración, existe consenso en torno a su proceso general. Por ejemplo, Thomson y Perry (2006) identifican un proceso de colaboración de tres fases: antecedentes, proceso de coordinación y resultados. Bryson, Crosby y Stone (2006) identifican el proceso con los mismos tres pasos, aunque resaltan que la estructura de gobernanza, las contingencias y las limitaciones de los actores afectarán el proceso. Asimismo, Wood y Gray (1991) describen un proceso también de tres fases: 1) precondiciones que hacen la colaboración posible y motivan a los actores a colaborar, 2) proceso a través del cual se logra la colaboración, y 3) resultados de la colaboración.

Figura 1. Esquema general para la colaboración

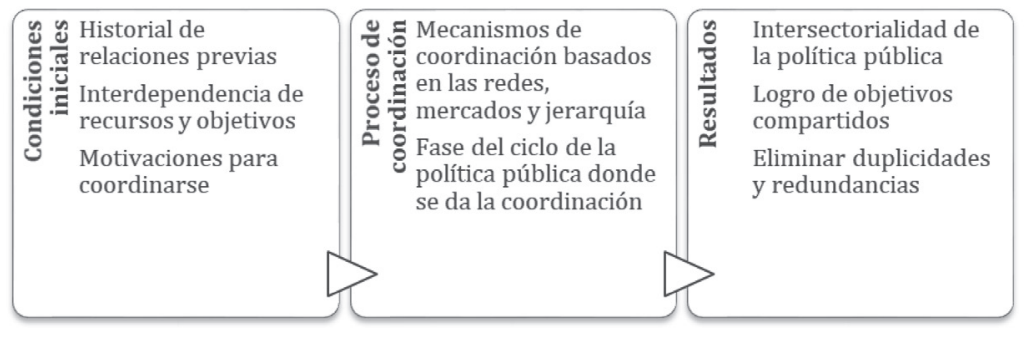

Fuente: elaboración propia con información de Bouckaert, Peters y Verhoest (2010); Bryson, Crosby y Stone (2006); Peters (2015); Thomson y Perry (2006); y Wood y Gray (1991).

Las condiciones iniciales del proceso de colaboración se refieren a la percepción de los involucrados de que el problema no puede ser resuelto por un solo actor y existe la necesidad de buscar la coordinación. Tal es el caso de muchos de los problemas vinculados a cuestiones sociales, como la pobreza o la inseguridad, que no pueden ser atendidos de forma aislada, por lo que la necesidad de colaboración o coordinación se vuelve evidente. El segundo factor de las condiciones iniciales es la historia previa de intercambios 
entre las organizaciones: si existen antecedentes de colaboraciones exitosas, el proceso de coordinación será más fácil; en contraparte, si existe una historia de competitividad y desconfianza, lograr la colaboración tomará más tiempo y será más complejo.

Por su parte, el proceso de coordinación es más complicado, ya que depende de las condiciones iniciales y de la fase en donde sucede la coordinación o colaboración, ya sea durante el diseño de una política pública o en el momento de su implementación. Es importante diferenciar la coordinación que se da durante la fase de diseño, ya que esta busca la integralidad de la política pública. Para Cunnill (2014), implica que "los sectores se pongan de acuerdo para actuar conjuntamente a fin de lograr un cambio social con respecto de la situación inicial" (p. 8). A diferencia de esto, la coordinación que se da durante la implementación de la política pública no busca la integralidad y cada actor persigue sus propios objetivos, por lo que la evaluación se basa en los resultados específicos del programa o secretaría, y no en el impacto de la problemática que la coordinación atiende.

Esta diferenciación es relevante porque la decisión sobre en qué momento se debe buscar la coordinación depende del objetivo que se persiga, es decir: si simplemente lo que se quiere es evitar la duplicidad y redundancia de las políticas públicas, la coordinación en la fase de la implementación puede ser la respuesta, pero si lo que se busca es mejorar el impacto en el bienestar de la población en pobreza, por ejemplo, idealmente la colaboración debe buscarse desde el diseño de la política pública, porque se reconoce que una sola dependencia de Gobierno no puede atacar por sí sola un problema multidimensional como la pobreza.

Finalmente, se necesita tener claro qué se busca con el proceso de coordinación, es decir, si es la intersectorialidad e integralidad de la política pública, entonces se requerirá un diseño en conjunto y un proceso de negociación mucho 
más largo y complejo, en donde los actores deberán ponerse de acuerdo y estar conscientes de su interdependencia y de que el resultado que piensan lograr en conjunto va más allá del que pueden lograr de forma individual. El actuar de forma coordinada a partir del diseño de la política pública disminuye, en cierta medida, la capacidad de rendir cuentas, esto debido a que la evaluación no se realiza con base en resultados particulares de cada secretaría, dependencia o programa, sino sobre los objetivos compartidos en los cuales se acordó (Peters, 2015). El caso contrario sucede cuando la coordinación se busca en la entrega del servicio en la fase de implementación de la política pública, donde básicamente lo que se logra es el acuerdo de disminuir las duplicidades y redundancias en la operación de los programas en lo individual.

De esta manera, se observa cómo coordinación y colaboración pueden ir de la mano y, lo que es más, que se requieren una y la otra ante problemas sociales como los que atiende la política social. Sin embargo, es importante señalar que coordinación y colaboración no son la respuesta a todos los problemas de la política pública, incluso, dada la complejidad que involucra la coordinación, se deben ponderar los posibles resultados frente a los costos para lograrla (Peters, 2015; Thomson y Perry, 2006; Bryson, Crosby y Stone, 2006).

\section{El Programa Nacional de Solidaridad (Pronasol)}

El Pronasol inició en 1988 bajo la presidencia de Carlos Salinas de Gortari (1988-1994). El nuevo Gobierno provenía de un proceso electoral muy controvertido, bajo la acusación de fraude electoral. Dresser (1997) afirma que el Pronasol fue una estrategia de gobernabilidad para evitar disturbios civiles y crear el consenso político necesario para la adopción de políticas económicas neoliberales. De acuerdo al consejo 
consultivo del Pronasol, de 1981 a 1987, la población que vivía en pobreza extrema en México pasó de 19.2\% (13.7 millones de personas) a 21.3\% (17.3 millones de personas), y en pobreza moderada de $25.8 \%$ (18.4 millones) a $29.5 \%$ (24 millones) (Consejo Consultivo del Programa Nacional de Solidaridad, 1991, p. 20). El Pronasol fue una iniciativa de la oficina presidencial y su administración se delegó a la Secretaría de Programación y Presupuesto, aunque el programa fue después transferido a la recién creada Secretaría de Desarrollo Social (Sedesol), en 1992.

A diferencia de todos los programas antipobreza anteriores, Pronasol estaba dirigido a toda la población en pobreza, rural y urbana (aproximadamente 41 millones de personas), pero especialmente a aquellos que vivían en pobreza extrema (17 millones) (Consejo Consultivo del Programa Nacional de Solidaridad, 1991, p. 54). El Pronasol se definió como "el instrumento creado por el Gobierno de la república para emprender una lucha frontal contra la pobreza extrema mediante la suma de esfuerzos coordinados en los tres niveles de Gobierno y los concertados con los grupos sociales" (Consejo Consultivo del Programa Nacional de Solidaridad, 1994, p. 8).

El objetivo del programa era mejorar el bienestar de la población ofreciendo medios productivos para la creación de un piso social básico (Ordóñez Barba, 2002). El programa estaba organizado en tres áreas: solidaridad para el bienestar social, solidaridad para la producción y solidaridad para el desarrollo regional.

Solidaridad para el bienestar social estaba conformado por programas de apoyo alimentario a través de tres mecanismos: 1) subsidios generales; 2) transferencias directas para pobres rurales y urbanos, a través de la distribución de vales de despensa y el acceso a alimentos básicos subsidiados a través de las tiendas de la Compañía Nacional de Subsistencias Populares (Conasupo); y 3) provisión de 
suplementos alimentarios y servicios de salud a los grupos más vulnerables, mujeres y niños.

Por su parte, solidaridad para la producción otorgaba préstamos para la promoción de actividades generadoras de ingreso, mientras que solidaridad para el desarrollo regional apoyaba la construcción de infraestructura básica en las comunidades pobres, principalmente respecto al acceso a agua potable, construcción de caminos y vivienda y el otorgamiento de títulos de propiedad (Campos y Vélez, 1994; Ordóñez Barba, 2002).

El 08 de diciembre de 1988, se aprobó el acuerdo presidencial que creó la Comisión del Programa Nacional de Solidaridad como órgano de coordinación y definición de las políticas, estrategias y acciones que en el ámbito de la administración pública se emprendieran para combatir los bajos niveles de vida, así como para asegurar el cumplimiento de los programas específicos que se instrumentaran (Consejo Consultivo del Programa Nacional de Solidaridad, 1991; 1994). La comisión estaba presidida por el titular del Ejecutivo federal e integrada por secretarios de Estado y los directores del IMSS, la Conasupo, el Instituto Nacional Indigenista, el Fondo Nacional para el Desarrollo de las Artesanías (Fonart), la cooperativa Fidepal, la Comisión Nacional de Zonas Áridas y la Comisión Nacional Forestal (Conafor), que a su vez formaba parte de la Subsecretaría de Desarrollo Regional, dependiente de la Secretaría de Programación y Presupuesto. Una vez que en 1992 se conformó la Secretaría de Desarrollo Social, el programa fue transferido a esta nueva dependencia.

Como un organismo auxiliar de las funciones de la comisión, se creó el consejo consultivo del Programa Nacional de Solidaridad, en el que estaban representados diversos grupos y sectores sociales que buscaban promover una mayor participación de la sociedad en el Pronasol. Se podían observar entre ellos representantes indígenas, campesinos, 
sectores populares, organizaciones no gubernamentales, sector privado e incluso académicos (Ordóñez Barba, 2002). También se creó el comité de evaluación, el cual tenía la tarea de realizar estudios sobre el impacto social y económico de los programas.

Pronasol, como el PIDER y la Coordinación General del Plan Nacional de Zonas Deprimidas y Grupos Marginados (Coplamar), promovía la coordinación entre niveles de Gobierno a través de los Comités de Planeación de Desarrollo Estatal (Coplade) y los convenios únicos de desarrollo (CUD), ${ }^{2}$ que eran instancias y mecanismos de planeación ya existentes. Asimismo, impulsaba la participación ciudadana por medio de la conformación de los comités comunitarios de Solidaridad, en los cuales se elegían representantes de las comunidades dentro de las asambleas promovidas por el programa, así como por medio de los consejos escolares de Solidaridad, organización de cobertura comunitaria de la cual existía una en cada escuela, con funciones de tipo operativo, ejecutivo, de administración y de control. De acuerdo al Consejo Consultivo del Programa Nacional de Solidaridad (1994), los consejos escolares representaban más de la mitad del total de los comités de Solidaridad de todo el país.

2. Los Coplade son un organismo público descentralizado del Gobierno estatal, dotados de personalidad jurídica y patrimonio propio, y tienen como funciones básicas: I) coordinar las relaciones entre los Gobiernos federal, estatal y municipal, derivadas de las acciones de planeación; 2) elaborar y actualizar el plan estatal de desarrollo; 3) proponer a los Gobiernos federal y estatal programas anuales de inversión para los estados y los municipios; y 4) evaluar los programas y acciones concertadas entre la federación y el estado. Los CUD (actualmente denominados convenios de desarrollo social), por su parte, son acuerdos de colaboración entre la federación y los estados por medio de los cuales se acuerdan compromisos jurídicos, financieros y programáticos para desarrollar las acciones previstas en los planes y programas de desarrollo. Un CUD contiene diversos programas, mediante los cuales la federación transfiere a los estados y municipios recursos suficientes para cumplir los compromisos derivados de los planes y programas de desarrollo, donde se conjunta la acción de los tres órdenes de Gobierno (Instituto Nacional de Administración Pública, 1985). 
De igual manera, se formaron comités municipales de Solidaridad, considerados "una figura puente para impulsar las demandas de las localidades hacia el nivel del municipio" (Consejo Consultivo del Programa Nacional de Solidaridad, 1994, p. 91). Estos servían para canalizar las demandas de los beneficiarios, pues se encargaban de integrar la lista de programas y proyectos que después era discutida en los Coplade. Los programas y proyectos aprobados eran incluidos posteriormente en los CUD entre la administración federal, los Gobiernos estatales y los municipios, donde se acordaban los montos a financiar. El Pronasol requería que parte del financiamiento para los programas y proyectos proviniera de los Gobiernos estatales y locales (Favela, 2003).

El programa contaba con una partida presupuestal específica, el llamado ramo XXVI: solidaridad y desarrollo regional, autorizada anualmente por la Cámara de Diputados. Esta se ejercía a través de los Gobiernos estatales y municipales y, ocasionalmente, a través de dependencias federales. El comité de Solidaridad era corresponsable de la programación-presupuestación, así como de la ejecución de la obra. Sólo se incorporaban al programa las obras que contaban con la participación y aceptación de la asamblea que había elegido al comité respectivo. Así, se establecían acciones corresponsables en el suministro de recursos, ejecución del trabajo y control de las obras determinadas (Consejo Consultivo del Programa Nacional de Solidaridad, 1994).

En 1994, después de que la administración del presidente Ernesto Zedillo (1994-2000) tomó el poder, México enfrentó otra crisis económica y el Pronasol fue eliminado gradualmente. El programa fue altamente criticado por diversas razones: sus efectos diluidos por su diversidad, sus altos costos, y su uso como mecanismo para la manipulación política que beneficiaba a aquellos que estaban mejor organizados, pero no necesariamente a los más necesitados (Favela, 2003; Guevara, 1997; Ordóñez Barba, 2002). El 
estudio de Guevara (1997) muestra que el presupuesto de gasto del Pronasol no estaba distribuido proporcionalmente con respecto a la población en pobreza. En algunos casos, los estados con los niveles más bajos de pobreza recibían los mismos recursos que aquellos estados con un porcentaje mucho mayor de población en pobreza.

\section{La Cruzada Nacional contra el Hambre}

El 01 de diciembre de 2012, el Partido Revolucionario Institucional (PRI) volvió a la Presidencia de México tras doce años de administraciones federales del Partido Acción Nacional (PAN). Ese día, el presidente de México estableció trece acciones para "mover a México" (su eslogan de administración), entre ellas la CNCH (Secretaría de Desarrollo Social, 2013b). Sin embargo, fue hasta enero de 2013 que se creó el Sistema Nacional para la Cruzada contra el Hambre (SNCH), que definió a la $\mathrm{CNCH}$ como una estrategia de inclusión y bienestar social que se implementaría a través de acciones coordinadas y participación social (Diario Oficial de la Federación, 2013a). Desde los primeros documentos, se reconoció que la Cruzada formaba parte de una política social de nueva generación encaminada a cumplir con la obtención de los derechos sociales para los mexicanos (Secretaría de Desarrollo Social, 2013b). Más tarde, como parte de esta nueva política social, el programa Oportunidades, representativo de la política social mexicana del siglo XXI, fue modificado para conformar el Programa de Inclusión Social Prospera (en adelante, Prospera).

De acuerdo con el Coneval (2015a), en México, para el 2012 la población en situación de pobreza extrema era del $9.8 \%$, y del $35.7 \%$ en pobreza moderada, con 2.4 carencias en promedio, siendo la de seguridad social la más presente (61.2\%), seguida por la alimentación (23.3\%). Ante esto, el problema de la alimentación se definió como el objeto 
de atención de la $\mathrm{CNCH}$, por lo que en 2013 sus principales objetivos fueron:

- Cero hambre a partir de una alimentación y nutrición adecuada de las personas en pobreza multidimensional extrema y carencia de acceso a la alimentación.

- Eliminar la desnutrición infantil aguda y mejorar los indicadores de peso y talla de la niñez. ${ }^{3}$

- Aumentar la producción de alimentos y el ingreso de los campesinos y pequeños productores agrícolas.

- Minimizar las pérdidas postcosecha y de alimentos durante su almacenamiento, transporte, distribución y comercialización.

- Promover la participación comunitaria para la erradicación del hambre (Diario Oficial de la Federación, 2013a, pp. 5-6).

El inicio de la estrategia fue accidentado, por lo que unos meses más tarde se hizo un relanzamiento de la misma. Los reajustes que se hicieron a la estrategia se incorporaron en el documento Programa Nacional México sin Hambre 20142018 (PNMSH), publicado el 30 de abril de 2014 en el Diario Oficial de la Federación. En este documento, se estableció la formalización de la $\mathrm{CNCH}$ y su ampliación territorial; además, se incorporó un objetivo más, relacionado con el impulso a la economía de los beneficiarios a través de la promoción del "desarrollo económico y el empleo de zonas de mayor concentración de pobreza extrema alimentaria" (Diario Oficial de la Federación, 2014, p. 2).

Durante el primer año de trabajo, su implementación se llevó a cabo en cuatrocientos municipios, pero con la declaración del PNMSH la CNCH se amplió a todo el territorio nacional, por lo que se proyectaba que, al finalizar 2015, la

3. En abril de 2014 , se cambió el verbo eliminar por disminuir en la definición de los objetivos de la estrategia. 
estrategia alcanzaría a toda la población objetivo (Diario Oficial de la Federación, 2014), definida esta como aquellas personas que se encuentran en situación de pobreza multidimensional extrema y carencia de acceso a la alimentación (Diario Oficial de la Federación, 2013a). De acuerdo con los primeros datos mostrados por Sedesol, se reconocía que en México 7.4 millones de personas se encontraban en esta situación. Más tarde, se reajustaría este dato y el PNMSH plantearía que existen 27.4 millones de personas en carencia de alimentación, de las cuales un poco más de 7 millones se encuentran en pobreza extrema alimentaria, conformando la población objetivo del programa (Diario Oficial de la Federación, 2014).

Esta estrategia se construyó dentro de lo que se ha denominado una política social de nueva generación, en la que los derechos sociales se establecen como punto de partida (Secretaría de Desarrollo Social, 2013b). En este sentido, se declara un nuevo enfoque de atención en el que la participación social, la revaloración del territorio y la coordinación interinstitucional se proponen como componentes esenciales del mismo (Diario Oficial de la Federación, 2014). Con base en esto, el PNMSH cuenta con cinco ejes: 1) participación social, 2) coordinación interinstitucional e intergubernamental, 3) territorialidad, 4) enfoque productivo, y 5) innovación y estrategia de cobertura (Diario Oficial de la Federación, 2014).

Nuevamente, la coordinación interinstitucional e intergubernamental es parte central de una política social. En el caso de la $\mathrm{CNCH}$, a través de ella, según el PNMSH, deben darse la vinculación de los programas y recursos a las comunidades, el impulso de un reordenamiento institucional que permita atender la pobreza extrema de alimentación y la promoción de la participación ciudadana (Diario Oficial de la Federación, 2014). Con la Cruzada se busca: 1) una mayor coordinación y concurrencia en la aplicación de los progra- 
mas sociales; 2) diálogo entre las dependencias federales que les permita unificar criterios; y 3) eficiente comunicación entre los tres órdenes de Gobierno; además, se espera que entre los resultados esté una mayor direccionalidad, evitar duplicidades, ordenar y coordinar las acciones públicas, a la par de "generar conjuntamente una política efectiva del ejercicio de los derechos sociales" (Diario Oficial de la Federación, 2014, p. 5).

Para la participación de las diferentes instituciones, organizaciones e incluso de la comunidad, se conformaron una serie de consejos y comités donde se incorporaron a diferentes actores en el diseño, el seguimiento, la implementación y la evaluación de la estrategia, el ya mencionado $\mathrm{SNCH}$. Dentro de las figuras federales enmarcadas en dicho sistema, se encuentran el comité de expertos, la comisión intersecretarial de la $\mathrm{CNCH}$ y un consejo consultivo, mientras que a nivel estatal y municipal se localizan los consejos consultivos estatales, las comisiones intersecretariales estatales y las comisiones intersecretariales municipales (Diario Oficial de la Federación, 2014).

Mención aparte requieren los comités comunitarios de la $\mathrm{CNCH}$, dado que una de las características de la estrategia es su lógica de implementación de abajo hacia arriba. Estos comités tienen la tarea de participar en el diagnóstico comunitario, mismo que servirá de insumo para la planeación de las acciones a seguir para lograr el desarrollo de la comunidad y que serán la base para darle seguimiento a las acciones llevadas por los diferentes programas e instituciones que participan en esta.

El consejo de expertos de la Cruzada contra el Hambre es definido como un órgano técnico-auxiliar del $\mathrm{SNCH}$, compuesto por académicos, investigadores y especialistas en pobreza y alimentación (Secretaría de Desarrollo Social, s/f; Diario Oficial de la Federación, 2014). Le compete presentar diagnósticos y propuestas tanto a la comisión intersecreta- 
rial y al consejo consultivo de la $\mathrm{CNCH}$ como a los comités estatales intersecretariales de la $\mathrm{CNCH}$.

La comisión intersecretarial para la instrumentación de la $\mathrm{CNCH}$, o comisión intersecretarial de la $\mathrm{CNCH}$, está integrada por representantes de diversas instituciones y secretarías federales. ${ }^{4}$ De acuerdo a las Normas de organización y funcionamiento interno de la comisión intersecretarial para la instrumentación de la Cruzada contra el Hambre, le corresponde proponer mecanismos de coordinación, analizar propuestas y aprobar mecanismos de evaluación y monitoreo, así como la creación de grupos de trabajo, opinar sobre proyectos de evaluación de resultados, aprobar informes anuales, proponer acciones "necesarias a las dependencias y entidades de la administración pública federal para garantizar la congruencia, transversalidad y sinergia entre programas y objetivos que integran la Cruzada" (Diario Oficial de la Federación, 2013b, p. 18), y proponer ajustes, entre otras encomiendas del Ejecutivo federal. Así, se estableció la conformación de grupos de trabajo que, de acuerdo al PNMSH, suman doce grupos (Diario Oficial de la Federación, 2014).

En el consejo nacional de la CNCH pueden participar los sectores público, privado y social dentro de la Cruzada. El consejo tiene la tarea de "mejorar y/o complementar las líneas de acción del programa" (Diario Oficial de la Federación, 2014, p. 10), y se integra por el secretario de Desa-

4. Secretaría de Desarrollo Social; Secretaría de Gobernación; Secretaría de Relaciones Exteriores; Secretaría de la Defensa Nacional; Secretaría de Marina; Secretaría de Hacienda y Crédito Público; Secretaría de Medio Ambiente y Recursos Naturales; Secretaría de Energía; Secretaría de Economía; Secretaría de Agricultura, Ganadería, Desarrollo Rural, Pesca y Alimentación; Secretaría de Comunicaciones y Transportes; Secretaría de Educación Pública; Secretaría de Salud; Secretaría del Trabajo y Previsión Social; Secretaría de Desarrollo Agrario, Territorial y Urbano; Secretaría de Turismo; Comisión Nacional para el Desarrollo de los Pueblos Indígenas; Instituto Nacional de las Mujeres y Sistema Nacional para el Desarrollo Integral de la Familia (Diario Oficial de la Federación, 20I3a, p. 6 ; Diario Oficial de la Federación, 2014). 
rrollo Social y representantes de organizaciones sociales y privadas, de instituciones académicas y organismos e instituciones internacionales (Diario Oficial de la Federación, 2013a), integrándose también a nivel estatal.

En el orden estatal, se establecieron los comités estatales intersecretariales, cuyo objetivo es generar mecanismos de coordinación entre dependencias de los tres órdenes de Gobierno. Estos están conformados por representantes de las secretarías federales, sus contrapartes estatales, así como por presidentes municipales y órganos de planeación municipales (Secretaría de Desarrollo Social, 2013a). Entre sus funciones se encuentran formular el programa estatal de trabajo y programas de inversión, gasto y financiamiento; operar los mecanismos de control, monitoreo y evaluación; así como la creación de grupos de trabajo locales y los informes trimestrales (Secretaría de Desarrollo Social, 2013a); asimismo, este comité tiene su contraparte municipal (Diario Oficial de la Federación, 2014).

Igualmente, el PNMSH propone una serie de instrumentos para la coordinación interinstitucional, como son las matrices de inversión, los padrones de derechohabientes sociales y la identificación de aquellos espacios donde se concentra mayormente la pobreza extrema de alimentación. En las matrices de inversión deben quedar plasmadas las obras y acciones que cada sector gubernamental -local y federalaportará. El padrón, por su parte, debe concentrar a los beneficiarios de los diferentes programas sociales de los tres órdenes de Gobierno. La identificación de la pobreza extrema de alimentación, finalmente, debe dar cuenta de la presencia institucional y mostrar las potencialidades y restricciones de las regiones (Diario Oficial de la Federación, 2014).

Los logros alcanzados en el 2014 muestran la participación de diversos actores tanto sociales como gubernamentales (ver: Tabla 2), particularmente a nivel federal, donde se 
pudieron integrar diversos esquemas de coordinación como el comité de expertos, así como la comisión intersecretarial y el consejo consultivo. En el caso de los niveles estatal y municipal, se presenta un panorama heterogéneo: en el caso de los comités estatales intersecretariales, se reportaron treinta y un comités, mientras que a nivel municipal se conformaron setecientos veintiocho comités municipales intersecretariales; sin embargo, para finales de 2014 eran 1012 los municipios donde se implementaba la $\mathrm{CNCH}, \mathrm{y}$ no se reportaba qué dinámica se había dado con aquellos municipios faltantes (Gobierno de la República, s/f).

Tabla 2. Acciones de coordinación de la $\mathrm{CNCH}$

\begin{tabular}{ll} 
Comités & Participan \\
Comité de expertos & 9 expertos \\
Comisión intersecretarial & 19 dependencias \\
& 90 programas \\
Consejo consultivo & Organizaciones de la sociedad civil \\
Comités estatales intersecretariales & 31 comités \\
Comités municipales & 728 comités \\
intersecretariales & \\
Comités comunitarios & 72000 \\
\hline
\end{tabular}

Fuente: elaboración propia con datos de Gobierno de la República (s/f) y Secretaría de Desarrollo Social (s/f).

Se cuenta con un padrón único de beneficiarios de la $\mathrm{CNCH}$, en el cual se concentra la información de los beneficiarios de ochenta y seis programas sociales (Gobierno de la República, s/f). De acuerdo a la página oficial del programa, para enero del 2016, el registro contabilizaba 4536275 personas atendidas por algún programa participante en la $\mathrm{CNCH}$ (Sinhambre, s/fa).

\section{6}




\section{De Pronasol a la $\mathrm{CNCH}$}

Ordóñez Barba (2002), a principios del siglo XXI, encontraba que los programas de atención a la pobreza no mostraban un cambio significativo en sus esquemas de intervención; acciones como la coordinación de las agencias federales y la incorporación de los Gobiernos locales son una práctica recurrente desde los años setenta.

$\mathrm{Al}$ hacer la comparación entre el Pronasol y la $\mathrm{CNCH}$, se encuentran una serie de semejanzas entre ambos, entre ellas sus esquemas de coordinación. Tomando como base las dimensiones de coordinación que propone Peters (2015), se puede identificar que, de aquellas con las que se cuenta con información, seis se presentan en ambos programas y se observan en la Tabla 3.

\section{Tabla 3. Dimensiones de coordinación en Pronasol y $\mathrm{CNCH}$}

\begin{tabular}{|c|c|c|}
\hline $\begin{array}{l}\text { Dimensión de } \\
\text { coordinación }\end{array}$ & Pronasol & $\mathrm{CNCH}$ \\
\hline Positiva/negativa & No hay información & No hay información \\
\hline $\begin{array}{l}\text { Para resultados/en el } \\
\text { proceso }\end{array}$ & Para resultados & Para resultados \\
\hline Vertical/horizontal & Ambos & Ambos \\
\hline $\begin{array}{l}\text { En el diseño/en la } \\
\text { implementación }\end{array}$ & $\begin{array}{l}\text { En la implementación; } \\
\text { aunque hay esquemas } \\
\text { de planeación, no se } \\
\text { puede hablar de diseño }\end{array}$ & $\begin{array}{l}\text { En la implementación; } \\
\text { aunque hay esquemas } \\
\text { de planeación, no se } \\
\text { puede hablar de diseño }\end{array}$ \\
\hline $\begin{array}{l}\text { Hacia adentro/hacia } \\
\text { afuera }\end{array}$ & Ambos & Ambos \\
\hline $\begin{array}{l}\text { Objetivos } \\
\text { similares/objetivos } \\
\text { complementarios }\end{array}$ & Ambos & Ambos \\
\hline $\begin{array}{l}\text { A corto plazo/a largo } \\
\text { plazo }\end{array}$ & En función del sexenio & En función del sexenio \\
\hline
\end{tabular}

Fuente: elaboración propia con base en las dimensiones identificadas por Peters (2015). 
Así, en el Pronasol, la coordinación por procesos o resultados tenía la intención de realizarse a partir del proceso político para la generación de la política pública, e incluso se señala que "Solidaridad comienza a operar con autonomía respecto de los procedimientos administrativos y los ritmos de algunas instituciones en las que predominan fuertes inercias burocráticas" (Consejo Consultivo del Programa Nacional de Solidaridad, 1994, p. 80).

El sustento de esta visión era que el programa operaba bajo la lógica de abajo hacia arriba, tomando como la principal unidad de acción el municipio-comunidad a través de la planeación participativa. De esta manera, el Pronasol atendía tres vertientes principales: bienestar social, producción y desarrollo regional, teniendo cada una de ellas sus propios programas específicos, ${ }^{5}$ que en total contabilizaban alrededor de treinta y seis programas.

Sin embargo, a pesar de que Pronasol operaba bajo una lógica de coordinación desde la implementación de la política pública, ya que esta se generaba de abajo hacia arriba, la evaluación y el desempeño de cada una de las vertientes y los programas que las conformaban se hicieron de forma aislada, es decir, por los resultados de cada programa en

5. Dentro de solidaridad para el bienestar, se ofrecían los programas de Infraestructura educativa, Solidaridad para una escuela digna, Programa de apoyo al servicio social, Niños en solidaridad, Hospital digno, Infraestructura de salud, Infraestructura hospitalaria, Centros de salud, Unidades médica rurales, Centro de bienestar social, Enfermeras en solidaridad, Maestros jubilados y pensionados, Jóvenes en solidaridad,Tenencia de la tierra, Solidaridad obrera,Agua potable rural y urbana, Alcantarillado rural y urbano, Electrificación, Urbanización, Vivienda, Infraestructura deportiva y Solidaridad forestal. Solidaridad para la producción lo conformaban los Fondos para la producción, Cajas de solidaridad, Empresas de solidaridad, Fondos regionales indígenas, Patrimonio cultural indígena, Mujeres en solidaridad, Apoyo a cafeticultores, Jornaleros agrícolas, Ecología productiva, Infraestructura de apoyo productivo, Apoyo a productores forestales, y otras acciones productivas. Solidaridad para el desarrollo regional incluía los programas de Fondos municipales Solidaridad y Carreteras y caminos rurales, esto sin incluir los programas de cien ciudades ni los dieciséis programas de desarrollo regional (Secretaría de Desarrollo Social y Programa Nacional de Solidaridad, 1994, pp. 29-30). 
específico, y no así desde el proceso mismo. En general, en la mayoría de los programas sociales, el discurso establecía que la intención era lograr la coordinación desde el proceso, pero fueron muy pocos los casos en que esto sucedió.

En el caso de la $\mathrm{CNCH}$, la coordinación se ubica también para los resultados cuando se busca dirigir las acciones de los diversos programas de los órdenes federal, estatal y municipal para evitar duplicidades. De acuerdo a Coneval (2015a), en la actualidad en México existen 6751 programas y acciones de desarrollo social implementados en los diferentes órdenes de Gobierno, reportándose doscientos treinta y tres en el orden federal, 3788 en el estatal y 2730 en el municipal (Coneval, 2015b). Los resultados esperados de los mismos son "una mayor direccionalidad y efectividad en extender el acceso a los derechos sociales" (Diario Oficial de la Federación, 2014, pp. 5). Así, se observa que en ambos programas los esfuerzos de coordinación han estado dirigidos a los resultados con el objetivo de eliminar las duplicidades, y no al proceso.

Siguiendo con las dimensiones de la coordinación propuestas por Peters (2015), los programas que conformaban cada una de las vertientes del Pronasol contaban con esquemas de coordinación tanto horizontales como verticales. Sin embargo, en el caso de Pronasol, el énfasis principal era la coordinación vertical con estados y municipios a través de mecanismos de participación ciudadana. Se señalaba que el municipio constituye la célula básica de la organización comunitaria y de vinculación del Estado con la sociedad (Consejo Consultivo del Programa Nacional de Solidaridad, 1994, p. 87).

La coordinación horizontal en Pronasol se buscó principalmente a través de la creación de la Sedesol, al afirmarse que "Con la creación de la Secretaría de Desarrollo Social se avanza hacia una reforma de instrumentos e instituciones. Se ha avanzado en la institucionalización del Programa 
Nacional de Solidaridad evitando su burocratización e imprimiéndole el perfil de política pública" (Consejo Consultivo del Programa Nacional de Solidaridad, 1994, p. 153). Además de la Sedesol, entidad responsable de gestionar y representar la institucionalidad de la política social, la coordinación horizontal se dio a partir de la Comisión del Programa Nacional de Solidaridad, que era presidida por el titular del Ejecutivo federal e integrada por secretarios de Estado y directores generales de diversas dependencias. Asimismo, se crearon también el consejo consultivo del Programa Nacional de Solidaridad, cuya intención era representar a los diversos grupos y sectores sociales, y el comité de evaluación del programa (Consejo Consultivo del Programa Nacional de Solidaridad, 1994, p. 123).

De la misma manera, dentro de la $\mathrm{CNCH}$ se presenta la coordinación horizontal a través del trabajo de las diferentes secretarías e instituciones federales que participan en ella. A esto, la estrategia lo denomina coordinación interinstitucional, en la que colaboran diecinueve dependencias (Gobierno de la República, s/f). La coordinación vertical, a su vez, se observa en la conformación de comités estatales y municipales, organismos a partir de los cuales se busca intercambiar información que permita enfocar de manera más eficiente las acciones de los diferentes órdenes de Gobierno. De acuerdo con la información oficial, entre 2013 y 2014 los comités sesionaron en ciento noventa y nueve ocasiones, con el objetivo de lograr concurrencia de acciones (Gobierno de la República, s/f).

A partir del análisis, es evidente que los mecanismos de coordinación tanto horizontales como verticales de ambos programas son una herramienta ampliamente usada en la política social mexicana, por lo que no se puede plantear esto como un objetivo novedoso en las acciones de combate a la pobreza de los Gobiernos mexicanos de los últimos años. Más bien, habrá que detenerse a revisar las razones por 
las cuales esto se sigue planteando como objetivo después de cuarenta años.

La dimensión por diseño o en la implementación tiene que ver en mayor medida con el proceso de política pública en donde se busca la coordinación, es decir, si es ex-ante, a partir de un diseño de arriba hacia abajo, o si es ex-post durante la implementación de la política pública tomando en cuenta la particularidades de cada caso. Esta se encuentra relacionada a la dimensión de proceso o de resultados (Peters, 2015); esta última se refiere a analizar si la coordinación se logra durante el proceso de diseño e implementación de una política pública, o bien, si como resultado se tuvo una política coordinada.

Pronasol contaba con una coordinación durante el diseño, específicamente para el caso de la construcción de obra pública en la vertiente de desarrollo regional. No obstante, para los otros programas que conformaban Pronasol, la coordinación se daba en la implementación y no desde el diseño.

En el caso de la $\mathrm{CNCH}$, la coordinación también se da en la implementación, y se hace evidente al observar el número de comités intersecretariales establecidos entre 2013 y 2014, y las sesiones de los mismos reportadas (Gobierno de la República, s/f), así como la firma de treinta y un acuerdos integrales para el desarrollo incluyente (Sinhambre, s/ fb). En la documentación del programa, se menciona que bajo el esquema de la $\mathrm{CNCH}$ el padrón de beneficiarios es compartido por los diferentes programas para evitar duplicidades, lo cual demuestra que la coordinación se da hasta la fase de implementación y no desde la fase del diseño de la política pública, dado que los diferentes programas ya estaban establecidos.

En relación a la dimensión de coordinación hacia adentro o hacia afuera (Peters, 2015), en los dos casos claramente se buscaron ambas. El Pronasol puso como centro a la participación de la comunidad para la operación de la política 
pública, y también se buscó la legitimación del programa a partir del consejo consultivo, además de que se utilizaron los mecanismos institucionalizados para la coordinación vertical, como los Coplade y los CUD, ya mencionados, y la creación de un comité intersecretarial en el primer nivel de Gobierno, esto último en cuanto a la coordinación horizontal. Este mismo modelo fue después replicado dentro del programa Progresa, pero con una mayor institucionalización, al conformarse una dependencia de coordinación nacional para el programa.

En el caso de la $\mathrm{CNCH}$, la coordinación hacia afuera se presenta con la incorporación de actores ajenos a las instituciones gubernamentales a través de esquemas de participación, como la firma de convenios con universidades y empresas privadas o, parte central de la estrategia, la participación social a través de los comités comunitarios, que también sucedía en Pronasol. Estas figuras pretenden hacer de los beneficiarios de los programas sociales individuos activos, capaces de dar seguimiento a las acciones de los diferentes órdenes de Gobierno que intervengan en su comunidad.

En relación a la dimensión de objetivos similares o complementarios de los programas que conformaban el Pronasol, existían algunos con objetivos complementarios. Una clara referencia de esto son las tres vertientes identificadas dentro del programa, ya que buscaban contribuir al bienestar de la población y la consecución del objetivo del programa de crear un piso social básico. Sin embargo, al interior de cada una de las vertientes, los programas que las conformaban tenían objetivos similares y complementarios. En el mismo sentido, la CNCH repite el objetivo de Pronasol de que la población más vulnerable pueda acceder a un piso básico de derechos consagrados en la Constitución. En el caso de Pronasol, esto se buscaba a través de la implementación de treinta y seis programas que perseguían tanto 
objetivos similares como complementarios, y en el caso de la $\mathrm{CNCH}$ se plantea a través de cincuenta y seis programas, eliminando las duplicidades donde algunos tienen objetivos similares y otros complementarios (Coneval, 2016). No obstante, sería necesario hacer un análisis más detallado de los objetivos de cada uno de los programas que conforman ambos programas para tener mayores elementos para argumentar sobre si esto ayudaba o dificultaba el proceso de coordinación.

Finalmente, si se toma la dimensión de Peters (2015) sobre el alcance del plazo -corto o largo- en la coordinación, se observa que, para el caso de ambos programas sería a largo plazo, ya que el alcance no se genera para la atención de una urgencia específica, sino para la operación e implementación de un programa social.

Sin embargo, históricamente en México, la vida de un programa social está vinculada al periodo presidencial en turno, es decir, al final de cada sexenio, la nueva administración deja de dar continuidad a los programas existentes y busca implementar su propia manera de hacer política. Incluso, en la actualidad hemos visto que el único programa social que subsiste y que ha logrado ser transexenal, aunque cada sexenio sufre de modificaciones tanto de nombre como de características, es Progresa-Oportunidades. Tal parece que los programas sociales son una herramienta poderosa de identificación presidencial. Obsérvese el cambio de nombre del programa, ya antes mencionado: durante la administración del presidente Zedillo surge Progresa, el cual sobrevive durante la administración de Vicente Fox denominándose Oportunidades, mantiene el nombre durante la administración de Felipe Calderón (aunque se le suman elementos), y, finalmente, es modificado como Prospera para la presidencia de Enrique Peña Nieto. 


\section{Conclusiones}

Como ya se comentó, la coordinación en programas sociales no es un tema nuevo en el caso de México. Sin embargo, se observa que no hay un reconocimiento de este proceso histórico en cada sexenio. Así, en la actual estrategia de desarrollo social, la $\mathrm{CNCH}$ promueve la coordinación como una forma de romper "con la idea del Gobierno archipiélago, de un Gobierno federal en el que cada dependencia y entidad definía sus propios objetivos" (Secretaría de Desarrollo Social, 2013b, p. 20), y continúa posicionando la búsqueda de la coordinación en la política pública como algo novedoso, ya que se establece que en iniciativas anteriores no se consideraban los aspectos positivos ni negativos, como las sinergias o duplicidades.

No obstante, a lo largo del análisis, se vuelve evidente que la coordinación ha sido un discurso recurrente en las diferentes administraciones federales, ya que, desde el primer programa de combate a la pobreza en este país, el PIDER, la búsqueda de la coordinación fue siempre un objetivo a lograr por parte de la política social. Incluso, muchos de los mecanismos de coordinación de la $\mathrm{CNCH}$ son similares a los implementados en los programas anteriores.

Si bien las dimensiones revisadas permiten reconocer las similitudes entre ambas estrategias analizadas, otra dimensión que puede dar luces sobre las formas en que se toman decisiones en las políticas públicas y que perfila elementos para analizar el éxito o fracaso de una propuesta de coordinación es el contexto político. Diversas investigaciones permiten reconocer la importancia de la política en la estructuración de las políticas públicas (Béland, 2005; Medellín, 2004; Schmidt, 2014), y nuevamente se observa esto en los casos analizados: ambos presidentes, Salinas de Gortari para el caso de Pronasol y Peña Nieto para el caso de la $\mathrm{CNCH}$, llegaron con una amplia oposición a sus Gobier- 
nos; en el caso de Salinas de Gortari, la oposición se dio en las calles, mientras que en el caso de Peña Nieto se observó en las nuevas tecnologías de la información, a través de las cuales se movilizaron ciertos sectores sociales. Ambos casos presentan a un Gobierno con una necesidad de credibilidad que debe atenderse, y una fórmula ya probada para ello es el trabajo con las comunidades y el impulso de la coordinación.

Finalmente, la reflexión más importante se encuentra en los problemas que la política social tiene, y específicamente la política de combate a la pobreza, para disminuir el número de pobres en el país. Los datos de Coneval (s/f) muestran que desde 1992, cuando se reportó que el 53.1\% de la población se encontraba en pobreza de patrimonio, y hasta la fecha, la pobreza sigue presentándose como un problema que atañe a más de la mitad de los mexicanos, a excepción del periodo comprendido del 2004 al 2008, durante el cual el valor más bajo fue de $42.9 \%$. Para el 2016 , el porcentaje de población en esta situación fue de $52.9 \%$ (Coneval, s/f).

La falta de coordinación ha sido recurrentemente utilizada como una justificante del fracaso de las políticas sociales. El presente análisis ha demostrado que la búsqueda de la misma no es un aspecto novedoso de la política social, sino una constante en los programas sociales implementados hasta la fecha. Incluso, se han mantenido los mismos mecanismos e instrumentos de coordinación al buscarla en la fase final, en los resultados y la implementación, y no se ha intentado romper con las inercias burocráticas al buscar la coordinación desde el proceso y desde el diseño mismo de la política pública.

Diversos autores han insistido sobre la necesidad de ponderar los beneficios frente a los costos de buscar la coordinación en la política pública (Peters, 2015; Thomson y Perry, 2006; Bryson, Crosby y Stone, 2006). Sin embargo, problemas complejos como la pobreza necesitan un abordaje 
multidimensional que involucre a los distintos sectores, ya que de lo contrario el énfasis recae en los indicadores de resultado (outputs) y no en el impacto (outcome), es decir, un cambio en la situación final. Lo anterior significaría que los beneficiarios de los programas sociales tendrán la posibilidad de disfrutar de los derechos sociales conferidos por la Constitución y romper el círculo vicioso de la pobreza. En este sentido, se hace referencia a la necesidad de un abordaje transversal como la única vía para lograr la integralidad de la política social.

Bibliografía

\section{6}


Brachet Márquez,V. (2004)."El Estado benefactor mexicano: Bibliografía nacimiento, auge y declive (1822-2002)", en J. Boltvinik, y A. Damián (coords.), La pobreza en México y el mundo realidades y desafios (Pp. 240-27). México: Siglo XXI.

Bryson,J.M., Crosby, B. C.,y Stone, M. M.(2006).“The Design and Implementation of Cross-Sector Collaborations: Propositions from the Literature". Public Administration Review, (66), 44-55. Recuperado de: http://onlinelibrary. wiley.com/doi/I0. I I I I/j. I540-62 I0.2006.00665.x/epdf Campos, E., yVélez, F. (1994).“La pobreza rural en México”, en F.Vélez, y E. Campos (eds.), La pobreza en México causas y políticas para combatirla (pp. 179-204). México: Fondo de Cultura Económica.

Coneval (s/f) Medición de la pobreza. Evolución de las dimensiones de la pobreza 1990-2016. Recuperado de: http:// www.coneval.org.mx/Medicion/Paginas/Evolucion-delas-dimensiones-de-pobreza.aspx

(20I5a). Resultados de pobreza en México 2014 a nivel nacional y por entidades federativas. Recuperado de: http://www.coneval.gob.mx/Medicion/MP/Paginas/ Pobreza_20I4.aspx

- (20I5b). Inventario nacional Coneval de programas y acciones de desarrollo social. Recuperado de: http://www. coneval.org.mx/Evaluacion/Paginas/inventario_nacional_de_programas_y_acciones_sociales.aspx

(2016). Balance de la Cruzada Nacional contra el Hambre 2013-20 I6. Recuperado de: http://www.coneval.org.mx/Evaluacion/ECNCH/Paginas/Balance_Cruzada_Nacional_contra_el_hambre.aspx

Consejo Consultivo del Programa Nacional de Solidaridad (I99I). El combate a la pobreza: lineamientos programáticos. México: El Nacional.

(I994). El Programa Nacional de Solidaridad. México: Fondo de Cultura Económica. 
Bibliografía
Cunnill Grau, N. (20I4).' 'La intersectorialidad en las nuevas políticas sociales. Un acercamiento analítico-conceptual". Gestión y Política Pública, 23(I), 4I. Recuperado de: http://www.scielo.org.mx/pdf/gpp/v23n l/v23n la l.pdf

Diario Oficial de la Federación (2013a). Decreto por el que se estalece el Sistema Nacional para la Cruzada contra el Hambre. México: Gobierno Constitucional de los Estados Unidos Mexicanos. Recuperado de: http:// www.sedesol.gob.mx/work/models/SEDESOL/Cruzada/I_DECRETO_POR_EL_QUE_SE_ESTABLECE_ EL_SISTEMA_NACIONAL_PARA_LA_CRUZADA_ CONTRA_EL_HAMBRE.pdf

(20I3b). Normas de organización y funcionamiento interno de la comisión intersecretarial para la instrumentación de la Cruzada contra el Hambre. México: Gobierno Constitucional de los Estados Unidos Mexicanos. Recuperado de:http://www.sedesol.gob.mx/work/models/SEDESOL/ Cruzada/2_NORMAS_DE_ORGANIZACION_Y_ FUNCIONAMIENTO_INTERNO_DE_LA_COMISION_INTERSECRETARIAL_PARA_LA_INSTRUMENTACION_DE_LA_CNCH.pdf

(2014). Decreto por el que se aprueba el Programa Nacional México sin Hambre 2014-2018. México: Gobierno Constitucional de los Estados Unidos Mexicanos. Recuperado de: http://www.dof.gob.mx/index. php? year $=2014 \&$ month $=04 \&$ day $=30$

Dresser, D. (1997). “En busca de la ligitimidad perdida”, en G. Martínez (ed.), Pobreza y política social en México (p. 2I). México: Fondo de Cultura Económica.

Favela, A. (2003). El combate a la pobreza en el sexenio de Zedillo. México: Universidad Autónoma Metropolitana Unidad Azcapotzalco.

Gobierno de la República (s/f). Plan Nacional de Desarrollo 20 I3-20 I 8. Programa Nacional México sin Hambre 20 I 42018. Logros 2014. Especial. México: Gobierno de la 
República. Recuperado de: https://www.gob.mx/cms/ Bibliografía uploads/attachment/file/27/39/20pe_nacional_mexicosinhambre.pdf

Gray, B. (1989). Collaborating: Finding common ground for multiparty problems. San Francisco: Jossey-Bass.

Guevara, S. A. (1997). "Programas de alivio de la pobreza en México: un ejercicio de evaluación”, en G. Martínez (ed.), Pobreza y política social en México (pp. 133-264). México: Fondo de Cultura Económica.

Instituto Nacional de Administración Pública (1985). Guía técnica núm. 10: la planeación del desarrollo municipal. Cuajimalpa: Instituto Nacional de Administración Pública. Levy, S. (1994). “La pobreza en México”, en F. Vélez, y E. Campos (eds.), La pobreza en México causas y políticas para combatirla (pp. I5-I I2). México: Fondo de Cultura Económica.

(2008). Good intentions, bad outcomes: Social policy, informality, and economic growth in Mexico. Washington: Brookings Institution Press.

— y Rodríguez, E. (2005). Sin herencia de pobreza: el programa Progresa-Oportunidades de México.WashingtonMéxico: Banco Interamericano de Desarrollo, Planeta.

Medellín, P. (2004). La política de las políticas públicas:propuesta teórica y metodológica para el estudio de las políticas públicas en países de frágil institucionalidad. Santiago de Chile: CEPAL. Recuperado de: http://repositorio.cepal. org/handle/I I 362/6082

O'Leary, R., y Vij, N. (2012). "Collaborative Public Management: Where Have We Been and Where Are We Going?". The American Review of Public Administration, 42(5), 507-522.

Ordóñez Barba, G. M. (2002). La política social y el combate a la pobreza en México. México: UNAM.

Peters, B. G. (1998). "Managing Horizontal Government: The Politics of Co-Ordination". Public Administration, 76, 
Bibliografía

295-3 I I. Recuperado de : http://onlinelibrary.wiley.com/ doi/I0.1 I I I/I467-9299.00 I02/epdf

(2005). “Concepts and theories of horizontal policy management" (documento presentado en el $x$ Congreso Internacional del CLAD sobre la Reforma del Estado y de la Administración Pública). Centro Latinoamericano de Administración para el Desarrollo (CLAD), Santiago, Chile.

(20I5). Pursuing horizontal management:The politics of public sector coordination. Kansas: University Press of Kansas.

Rogers, D. L., y Whetten, D. A. (1982). Interorganizational coordination:Theory, research, and implementation. Ames: lowa State University Press.

Schmidt, V. (20I4). La política de los pactos sociales y la protección social: ¿importa el discurso? Experiencias internacionales. Santiago de Chile: CEPAL. Recuperado de: https://www.cepal.org/es/publicaciones/35902-lapolitica-pactos-sociales-la-proteccion-social-importadiscurso-experiencias

Secretaría de Desarrollo Social (s/f). Lineamientos de organización y funcionamiento del consejo de expertos de la Cruzada contra el Hambre. México:Sedesol. Recuperado de: http://www.sedesol.gob.mx/work/models/sedesol/ cruzada/9_comite_de_expertos.pdf

(20I3a). Comités estatales intersecretariales: lineamientos básicos. México: Sedesol. Recuperado de: https:// www.gob.mx/cms/uploads/attachment/file/I 07974/I I LINEAMIENTOS_BASICOS_DE_LOS_COMITES ESTATALES_INTERSECRETARIALES.pdf

(20I3b). Política social de nueva generación y Cruzada Nacional contra el Hambre: documento de trabajo.Versión I.I. México: Sedesol. Recuperado de. http://www. sedesol.gob.mx/work/models/SEDESOL/PDF/POLITICA_SOCIAL_DE_NG_Y_CNCH.pdf 
y Programa Nacional de Solidaridad (1994). Solidaridad: seis años de trabajo. México: Sedesol.

Sinhambre $(\mathrm{s} / \mathrm{fa})$. Personas en condiciones de pobreza extrema de alimentación que son atendidas por la $\mathrm{CNCH}$. Recuperado de:http://sinhambre.gob.mx/personas_en_condiciones de_pobreza_extrema_de_alimentacion_que_son_atendidas_por_la_cnch/

(s/fb). Recuperado de: http://sinhambre.gob.mx/ transparencia/page/3/

Thompson, G.,y Open University ( 1991). Markets, hierarchies, and networks:The coordination of social life. Londres: Sage Publications.

Thomson,A. M., y Perry, J. L. (2006). “Collaboration Processes: Inside the Black Box". Public Administration Review, (66), 20-32.

Vilas, C. (2000). “Más allá del 'Consenso de Washington'. Un enfoque desde la política de algunas propuestas del Banco Mundial sobre reforma institucional". Revista CLAD Reforma y Democracia, ( 8). Recuperado de: http:// old.clad.org/portal/publicaciones-del-clad/revista-cladreforma-democracia/articulos/0 /8-octubre-2000/ bfmas-alla-del-consenso-de-washington-un-enfoquedesde-la-politica-de-algunas-propuestas-del-bancomundial-sobre-reforma-institucional-I

Wood, D. J., y Gray, B. (199I). "Toward a Comprehensive Theory of Collaboration". The Journal of Applied Behavioral Science, 27(2), I39-I62.

Bibliografía 